\title{
Braid Group, Knot Theory and Statistical Mechanics, II
}




\author{
Editors-in-Charge \\ H Araki (RIMS, Kyoto) \\ $\checkmark \mathrm{G} \mathrm{Kac}(M I T)$ \\ D H Phong (Columbia University) \\ S-T Yau (Harvard University)

\section{Associate Editors} \\ L Alvarez-Gaumé (CERM) \\ J P Bourguignon (Ecole Polytechnique, Palaiseau) \\ T Eguchi (University of Tokyo) \\ B Julia (CNRS, Paris) \\ F Wilczek (Institute for Advanced Study, Princeton)
}

\title{
Published
}

Vol. 1: Mathematical Aspects of String Theory edited by S-T Yau

Vol. 2: Bombay Lectures on Highest Weight Representations of Infinite Dimensional Lie Algebras by V G Kac and A K Raina

Vol. 3: Kac-Moody and Virasoro Algebras: A Reprint Volume for Physicists edited by $P$ Goddard and $D$ Olive

Vol. 4: Harmonic Mappings, Twistors and $\sigma$-Models edited by $P$ Gauduchon

Vol. 5: Geometric Phases in Physics edited by A Shapere and F Wilczek

Vol. 7: Infinite Dimensional Lie Algebras and Groups edited by V Kac

Vol. 8: Introduction to String Field Theory by W Siegel

Vol. 9: Braid Group, Knot Theory and Statistical Mechanics odited by $C N$ Yang and $M L G e$

Vol. 10: Yang-Baxter Equations in Integrable Systems edited by $M$ Jimbo

Vol. 11: New Developments in the Theory of Knots edited by T Kohno

Vol. 12: Soliton Equations and Hamiltonian Systems by L A Dickey

Vol. 14: Form Factors in Completely Integrable Models of Quantum Field Theory by F A Smimov

Vol. 15: Non-Perturbative Quantum Field Theory - Mathematical Aspects and Applications Selected Papers of Jürg Fröhlich

Vol. 16: Infinite Analysis - Proceedings of the RIMS Research Project 1991 edited by A Tsuchiya, T Eguchi and M Jimbo 
Advanced Series in Mathematical Physics

Vol. 17

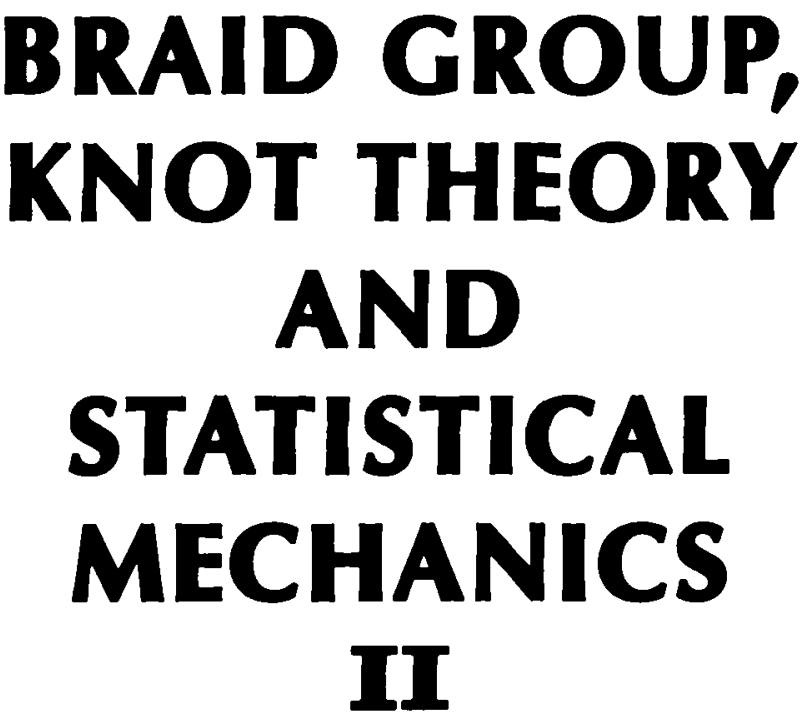

Editors

C. N. Yang

Department of Physics

State University of New York, Stony Brook

\author{
M. L. Ge \\ Institute of Mathematics \\ Nankai University
}




\section{Published by}

World Scientific Publishing Co. Pte. Ltd.

P O Box 128, Farrer Road, Singapore 9128

USA office: Suite 1B, 1060 Main Street, River Edge, NJ 07661

UK office: 73 Lynton Mead, Totteridge, London N20 8DH

\section{BRAII GROUP, KNOT THEORY AND STATISTICAL MECHANICS, I}

Copyright $\odot 1994$ by World Scientific Publishing Co. Pte. Ltd.

All rights reserved. This book, or parts thereof, may not be reproduced in any form or by any means, electronic or mechanical, including photocopying, recording or any information storage and retrieval system now known or to be invented, without written permission from the Publisher.

For photocopying of material in this volume, please pay a copying fee through the Copyright Clearance Center, Inc., 27 Congress Street, Salem, MA 01970, USA.

ISBN 981-02-1524-X

Printed in Singapore by Utopia Press. 


\section{FOREWORD}

It has been four years since the publication in 1989 of the previous volume bearing the same title as the present one. An enormous amount of work has been done in the meantime. We hope the present volume will provide a summary of some of these works which are still progressing in several directions.

Chen Ning Yang

Mo-Lin $\mathrm{Ge}$ 


\section{CONTENTS}

Foreword

On the Combinatorics of Vassiliev Invariants

J. S. Birman

Solvable Models, Link Invariants and Their Applications

to Physics

T. Deguchi, M. Wadati

The Yang-Baxter Symmetry in Field Theory

H. J. De Vega

Quantum Symmetry in Conformal Field Theory by Hamiltonian Methods

L. D. Faddeev

Yang-Baxterization and Algebraic Structures

M. L. Ge, K. Xue, Y. S. Wu

Introduction to the Yang-Baxter Equation

153

M. Jimbo

Notes on Subfactors and Statistical Mechanics

$V . F$. R. Jones

Polynomial Invariants in Knot Theory

202

L. H. Karffman

Spin Networks, Topology and Discrete Physics

L. H. Kauffman

Tunnel Numbers of Knots and Jones-Witten Invariants

T. Kohno

Symmetry Approach to Solvable Lattice Models

T. Miwa 
Algebras of Loops on Surfaces, Algebras of Knots, and Quantization

V. G. Turaev

Quantum Field Theory and the Jones Polynomial

E. Witten

Knot Invariants and Statistical Mechanics: A Physicist's Perspective

F. $Y . W$ w 